\title{
Physical Vapour Deposited Biomedical Coatings
}

\author{
Bryan W. Stuart ${ }^{1, *}$ and George E. Stan $2, *$ D \\ 1 Department of Materials, University of Oxford, Parks Road, Oxford OX1 3PH, UK \\ 2 National Institute of Materials Physics, RO-077125 Magurele, Romania \\ * Correspondence: bryan.stuart19@gmail.com (B.W.S.); george_stan@infim.ro (G.E.S.); \\ Tel.: +40-21-241-8128 (G.E.S.)
}

\begin{abstract}
This Special Issue was devoted to developments made in Physical Vapour Deposited (PVD) biomedical coatings for various healthcare applications. The scrutinized PVD methods were RadioFrequency Magnetron Sputtering (RF-MS), Cathodic Arc Evaporation, Pulsed Electron Deposition and its variants, Pulsed Laser Deposition, and Matrix Assisted Pulsed Laser Evaporation (MAPLE), due to their great promise especially in the dentistry and orthopaedics. These methods have yet to gain traction for industrialization and large-scale application in biomedicine. A new generation of implant coatings can be made available by the (1) incorporation of organic moieties (e.g., proteins, peptides, enzymes) into thin films by innovative methods such as combinatorial MAPLE, (2) direct coupling of therapeutic agents with bioactive glasses or ceramics within substituted or composite layers via RF-MS, or (3) by innovation in high energy deposition methods such as arc evaporation or pulsed electron beam methods.
\end{abstract}

Keywords: physical vapour deposition; thin-films; medical devices; bioactivity; biomimicry

Citation: Stuart, B.W.; Stan, G.E. Physical Vapour Deposited Biomedical Coatings. Coatings 2021, 11, 619. https://doi.org/10.3390/ coatings11060619

Received: 7 May 2021

Accepted: 18 May 2021

Published: 21 May 2021

Publisher's Note: MDPI stays neutral with regard to jurisdictional claims in published maps and institutional affiliations.

Copyright: (c) 2021 by the authors. Licensee MDPI, Basel, Switzerland. This article is an open access article distributed under the terms and conditions of the Creative Commons Attribution (CC BY) license (https:// creativecommons.org/licenses/by/ $4.0 /)$.
As research and development into medical devices reaches an all-time high, surface functionalization through less invasive, low dimensional thin-film coatings is at the forefront of optimising bio-integration, bio-activation, and biomechanics. In the last decades, physical vapour deposition (PVD) techniques have gained attraction for their diverse capabilities in blending and manufacturing highly adhesive, novel materials with minimally invasive thicknesses from ångströms to the micrometres scale.

It has long been the opinion of the industrial community that technologies based on thermal spray technologies (e.g., plasma, flame, detonation, cold, high-velocity atmospheric, high-velocity oxy-fuel, and high-velocity suspension flame spraying) and solution-based methods (e.g., sol-gel, solution casting) provide high throughput capabilities and low start-up costs suitable for commercial scale manufacturing. However, as the precision, versatility, scale, and accessibility of standardized and scaled up PVD systems grows, the medical device industry will seek advanced biomaterial applications for the next generation of functional layers with long-term reliability and high success rates.

This Special Issue of Coatings features five full-length articles, all pertaining to the field of bio-functional surfaces with dentistry and orthopaedic applications, spanning from (i) silicon highly doped hydroxyapatite [HA, $\mathrm{Ca}_{10}\left(\mathrm{PO}_{4}\right)(\mathrm{OH})_{2}$ ] coatings [1] and alkali-free copper and gallium co-doped silica bioactive glass [2] layers deposited onto titanium (Ti)based substrates by Radio-Frequency Magnetron Sputtering (RF-MS), for osseointegration and /or antibacterial uses; to (ii) thin films of HA on polyletheretherketone (PEEK) fabricated by RF-MS [3]; (iii) biological derived HA coatings doped with lithium carbonate or lithium phosphate synthesized by Pulsed Laser Deposition on a 3D printed Ti implants [4]; and to (iv) carbonitride coatings of TiCN, TiSiCN realized by cathodic arc deposition method aiming to improve the wear resistance of the $\mathrm{CoCr}$ alloys often used for joint endoprosthetic fabrication [5]. The biofunctionality of the implant-type coatings was surveyed by an array of in vitro (corrosion investigations in biomimetic medium [5], cytocompatibil- 
ity tests [1,2] and antimicrobial efficacy [2]) and in vivo (implantation into rabbit femoral condyles, followed by mechanical removal at 4 and 9 weeks [4]) biological assays.

Furthermore, this Special Issue suitably demonstrates the current landscape for future generations of biological layers containing two extensive review articles on the emerging popularity of more recent/novel manufacturing methods and their relevance to biomaterial applications. These specifically pertain to the Pulsed Electron Deposition (PED) [6] and Combinatorial Laser deposition technologies [7].

PED is a spark discharge ablation method which accelerates a high energy electron beam towards a target. The functionality has changed over time and most recent developments extract the electrons from a generated plasma in a dielectric tube, termed Pulsed Plasma Deposition (PPD). In an additional development step, ionized jet deposition (IJD), utilizes gas jet entrainment and short pulses of up to megawatts. PPD and IJD exhibit high electrical efficiencies of $30 \%$ and $88 \%$, respectively, with higher deposition rates leading to lower capital costs. Of great relevance in current orthopaedic coatings, the high voltage potentials (up to $25 \mathrm{kV}$ ) enable deposition of the full spectrum from amorphous transparent film to high energy crystalline phases in a single-step, demonstrated to produce layers such as bone-like HA capable of stimulating osseointegration. Liquori et al. [6] review the application of PED, PPD, and IJD for materials applicable in orthopaedic prosthetics such as wear resistant crystalline yttria-stabilized zirconia coatings or bioactive strontium doped-HA, Bioglass ${ }^{\circledR} 45 S 5$, nanostructured silver, and HA-magnetite composite layers onto ultra-high-molecular-weight polyethylene, titanium alloys, stainless steel, glass, silicon, and PEEK substrates.

Axente et al. [7] reviews PLD-based techniques (well-known for preservation of stoichiometry and tune-ability of input energies for desired chemistries/reactability with working gases (e.g., for the deposition of TiN coatings)). The state-of-the-art manufacturing development is described, with emphasis on the Matrix Assisted Pulsed Laser Evaporation (MAPLE) method's capability to fabricate thin (heat-sensitive) organic, inorganic, and hybrid organic-inorganic films. MAPLE relies on UV laser irradiation of a cryogenic target and enables the safe transfer of proteins, biopolymers, enzymes, or polysaccharides, as well as more conventional deposits of calcium phosphates, from a target to a substrate. Some applications include deposition of fibronectin, bovine serum albumin-functionalized graphene oxide nanomaterials. Axente et al. [7] focuses on the "Combinatorial" approach, referring to the use of multiple laser systems to produce material blends across a substrate, demonstrating formation of strontium doped HA-zinc doped $\beta$-tricalcium phosphate in bone regenerative applications, sulphated Halomonas Levan blended quaternized low molecular weight chitosan for antibacterial properties, graphene oxide nanomaterials with hybrid bovine serum albumin, and introduced Dabrafenib/Trichostatin A drugs for targeting melanoma cells.

As presented within, PVD modified implant surfaces benefit from enhanced bioactivity, leading to advantageous cell-surface interactions to assist in regenerating tissue. The Special Issue successfully draws attention towards a wide range of PVD methods and novel applications which hitherto have not gained traction for industrialization yet possess enormous benefit for the world of medical care. The methods shown here-RadioFrequency Magnetron Sputtering, Cathodic Arc Evaporation, Pulsed Electron Deposition and its variants, Pulsed Laser Deposition, and Matrix Assisted Pulsed Laser Evaporationdemonstrated feasible application and great promise as strong advocates for the future widespread adoption of PVD films in dentistry and orthopaedics. A broader field of biomaterials can now be tackled through incorporation of proteins, peptides, enzymes into implant coatings via novel methods such as MAPLE or by the direct coupling of therapeutic agents with bioactive glasses or ceramics within substituted or composite layers via the sputter deposition variants.

Author Contributions: Conceptualization, B.W.S. and G.E.S.; writing—original draft preparation, B.W.S. and G.E.S.; writing—review and editing, B.W.S. and G.E.S.; funding acquisition, G.E.S. Both authors have read and agreed to the published version of the manuscript. 
Funding: This work was funded by Romanian National Authority for Scientific Research and Innovation (CNCS-UEFISCDI) in the framework of projects PN-III-P1-1.1-TE-2016-1501 and PN-IIIP1-1.1-TE-2019-0463.

Institutional Review Board Statement: Not applicable.

Informed Consent Statement: Not applicable.

Conflicts of Interest: The authors declare no conflict of interest.

\section{References}

1. Coe, S.C.; Wadge, M.D.; Felfel, R.M.; Ahmed, I.; Walker, G.S.; Scotchford, C.A.; Grant, D.M. Production of high silicon-doped hydroxyapatite thin film coatings via magnetron sputtering: Deposition, characterisation, and in vitro biocompatibility. Coatings 2020, 10, 190. [CrossRef]

2. Stan, G.E.; Tite, T.; Popa, A.C.; Chirica, I.M.; Negrila, C.C.; Besleaga, C.; Zgura, I.; Sergentu, A.C.; Popescu-Pelin, G.; Cristea, D.; et al. The beneficial mechanical and biological outcomes of thin copper-gallium doped silica-rich bio-active glass implant-type coatings. Coatings 2020, 10, 1119. [CrossRef]

3. Hussain, S.; Rutledge, L.; Acheson, J.G.; Boyd, A.R.; Meenan, B.J. The surface characterisation of polyetheretherketone (Peek) modified via the direct sputter deposition of calcium phosphate thin films. Coatings 2020, 10, 1088. [CrossRef]

4. Duta, L.; Neamtu, J.; Melinte, R.P.; Zureigat, O.A.; Popescu-Pelin, G.; Chioibasu, D.; Oktar, F.N.; Popescu, A.C. In vivo assessment of bone enhancement in the case of $3 \mathrm{~d}$-printed implants functionalized with lithium-doped biological-derived hydroxyapatite coatings: A preliminary study on rabbits. Coatings 2020, 10, 992. [CrossRef]

5. Dinu, M.; Pana, I.; Scripca, P.; Sandu, I.G.; Vitelaru, C.; Vladescu, A. Improvement of CoCr alloy characteristics by Ti-based carbonitride coatings used in orthopedic applications. Coatings 2020, 10, 495. [CrossRef]

6. Liguori, A.; Gualandi, C.; Focarete, M.L.; Biscarini, F.; Bianchi, M. The pulsed electron deposition technique for biomedical applications: A review. Coatings 2020, 10, 16. [CrossRef]

7. Axente, E.; Sima, L.E.; Sima, F. Biomimetic coatings obtained by combinatorial laser technologies. Coatings 2020, $10,463$. [CrossRef] 